\title{
A CLASS OF COMPLETE ORTHOGONAL SEQUENCES OF BROKEN LINE FUNCTIONS
}

\author{
BY \\ J. L. SOX, JR.
}

\begin{abstract}
A class of orthonormal sets of continuous broken line functions is defined. Each member is shown to be complete in $L_{2}(0,1)$ and pointwise convergence theorems are obtained for the Fourier expansions relative to these sets.
\end{abstract}

1. Introduction. It was shown in [2] that each sequence of points which is dense in $[0,1]$ determines a complete orthonormal set of step functions in $L_{2}(0,1)$. In this paper we prove that each such sequence of points also determines a complete orthonormal set of continuous broken line functions similar to that constructed by Franklin [1]. The Fourier expansion of a function $f \in L_{2}(0,1)$ relative to a set of this class is found to converge at each point of continuity of $f$ and is shown to converge uniformly on $[0,1]$ when $f$ is continuous on this interval.

2. Definitions. Suppose that $A=\left\{a_{n}\right\}_{n=1}^{\infty}$ is a sequence of distinct points in $(0,1)$ which is dense in $[0,1]$ and let $\left\{h_{n}\right\}_{n=0}^{\infty}$ be the set of linear functions defined by

$$
\begin{array}{rlrl}
h_{0}(x) \equiv 1, & h_{1}(x)=x, & x \in[0,1] ; \\
h_{n+1}(x)= & 0, & x \in\left[0, a_{n}\right), \\
= & x-a_{n}, & & x \in\left[a_{n}, 1\right] .
\end{array}
$$

Since it is evident that no $h_{i}$ is a linear combination of the other functions in the set, we see that the $h_{i}$ are linearly independent on $[0,1]$. Thus, one can employ the Gram-Schmidt process to construct an orthonormal sequence $\left\{u_{n}(x)\right\}$ such that each $u_{n}$ is a linear combination of the $h_{i}, i \leqq n$. Because of the triangular nature of this construction, each $h_{n}$ can also be expressed as a linear combination of the $u_{i}$, $i \leqq n$.

3. Completeness of $\left\{u_{n}\right\}$. To prove that the sequence of functions $\left\{u_{n}\right\}$ is complete in $L_{2}(0,1)$, one needs an obvious property of the sequence $A$ which is given in Lemma 1. In this lemma and throughout this paper the term "adjacent points" of a finite subset $A_{N} \subset A$ will be used to denote successive elements of the subset when its elements are arranged in order of magnitude; i.e. $a_{m}$ and $a_{n}$ are adjacent points of $A_{N}$ if and only if there is no $a_{k} \in A_{N}$ such that $a_{m}<a_{k}<a_{n}$ or $a_{n}<a_{k}<a_{m}$.

Received by the editors July 6, 1971.

AMS 1969 subject classifications. Primary 4211, 4215, 4216, 4217; Secondary 4020.

Key words and phrases. Franklin functions, Fourier series, orthonormal set of functions, complete in $L_{2}(0,1)$, pointwise convergence. 
LEMMA 1. Let $A=\left\{a_{1}, a_{2}, \ldots\right\}$ be a sequence of distinct points of $(0,1)$ which is dense in $[0,1]$. Then for each $\delta>0$ there is an integer $N_{\delta}$ such that if $N>N_{\delta}$, (i) any pair of adjacent points $a_{m}$ and $a_{n}$ in the subset $A_{N}=\left\{a_{1}, a_{2}, \ldots, a_{N}\right\}$ satisfy $\left|a_{m}-a_{n}\right|$ $<\delta$; (ii) $d\left(x, A_{N}\right)<\delta$ for $x \in[0,1] .\left(d\left(x, A_{N}\right)\right.$ is the distance from $x$ to the $A_{N}$ defined in the usual manner.)

THEOREM 1. The orthonormal sequence of functions $\left\{u_{n}\right\}$ is complete in $L_{2}(0,1)$.

Proof. Let $0<a_{i_{1}}<a_{i_{2}}<\cdots<a_{i_{N}}<1$ be the points of $\left\{0, a_{1}, a_{2}, \ldots, a_{N}, 1\right\}$ arranged in order of magnitude. If $P_{N}$ is any continuous polygonal function (broken line function) which is linear on each subinterval $\left[a_{i_{k-1}}, a_{i_{k}}\right]$ of the partition of $[0,1]$ determined by these points, it is clear that $P_{N}$ can be expressed as a linear combination of the $h_{i}, i \leqq N$. Thus since each $h_{i}, i \leqq N$, is a linear combination of the $u_{i}, i \leqq N$, any such $P_{N}$ is a linear combination of the $u_{i}, i \leqq N$.

Now suppose that $F$ is any continuous function on $[0,1]$ and let $\delta$ be a positive number such that $\left|F\left(x_{1}\right)-F\left(x_{2}\right)\right|<\varepsilon / 2$ when $x_{1}, x_{2} \in[0,1]$ and $\left|x_{1}-x_{2}\right|<\delta$. By Lemma 1 we can choose an integer $N_{\delta}$ such that if $N>N_{\delta}$, the norm of the partition of $[0,1]$ determined by the points of $A_{N}$ is less than $\delta$. Therefore, the broken line function $P_{N}$ which equals $F$ at each point of this partition and is linear elsewhere in $[0,1]$ satisfies $\left|P_{N}(x)-F(x)\right|<\varepsilon$ for $x \in[0,1]$. It follows from the preceding remarks that there is a linear combination of $u_{i}, i \leqq N$, say $T_{N}$, such that $\left|T_{N}(x)-F(x)\right|<\varepsilon$ if $x \in[0,1]$ or such that

$$
\left\|T_{N}-F\right\|_{2}^{2}=\int_{0}^{1}\left[T_{N}-F\right]^{2} d x<\varepsilon^{2} .
$$

Since the set of continuous functions on $[0,1]$ is dense in $L_{2}(0,1)$, we conclude from the last inequality that the set of linear combinations of the $u_{i}$ is also dense in this space. This statement, of course, implies that the sequence $\left\{u_{n}\right\}$ is complete in $L_{2}(0,1)$.

4. Convergence of the Fourier $\left\{u_{n}\right\}$ expansion. Since $\left\{u_{n}\right\}$ is a complete orthonormal sequence in $L_{2}(0,1)$, each $f \in L_{2}(0,1)$ has the norm-convergent Fourier expansion

$$
f(x) \sim \sum c_{k} u_{k}(x)
$$

where

$$
c_{k}=\int_{0}^{1} f u_{k} d x
$$

We next investigate the pointwise convergence of this expansion.

THEOREM 2. The Fourier $-u_{n}$ expansion of $f \in L_{2}(0,1)$ converges to $f(x)$ at each point $x \in[0,1]$ at which $f$ is continuous.

Proof. Let $S_{N}(x, f)$ denote the $N$ th partial sum of (1). Since each $u_{i}$ is a linear 
combination of the $h_{k}, k \leqq i, S_{N}$ itself is a linear combination of the $h_{i}, i \leqq N$, and thus is a continuous broken line function which is linear on each subinterval of the partition of $[0,1]$ determined by the points of $A_{N}=\left\{a_{1}, a_{2}, \ldots, a_{N}\right\}$. Suppose $0<a_{i_{1}}<a_{i_{2}}<\cdots<a_{i_{N}}<1$ are the points of $A_{N}$ arranged in order of magnitude and let $K_{0}, K_{1}, \ldots, K_{N}$ denote the characteristic functions of the intervals [0, $a_{i_{1}}$ ), $\left[a_{i_{1}}, a_{i_{2}}\right), \ldots,\left[a_{i_{N}}, 1\right]$. Then

$$
S_{N}(x, f)=\sum_{0}^{N} c_{i} u_{i}=\sum_{0}^{N}\left(\alpha_{i}+\beta_{i} h_{i}\right) K_{i}
$$

where the $\alpha$ 's and $\beta$ 's are constants. To determine $\alpha_{i}$ and $\beta_{i}$ we use the well-known fact that if $T_{N}$ is any linear combination of the $u_{i}, i \leqq N, \int_{0}^{1}\left(f-T_{N}\right)^{2} d x$ assumes its minimum value when $T_{N}=S_{N}$. Thus $\alpha_{i}$ and $\beta_{i}$ must have values which minimize

$$
\int_{0}^{1}\left[f-\sum_{0}^{N}\left(\alpha_{i}+\beta_{i} h_{i}\right) K_{i}\right]^{2} d x
$$

and when the partial derivatives of this integral with respect to $\alpha_{m}$ and $\beta_{m}$ are equated to 0 , one has for each $m=0,1,2, \ldots, N$,

$$
\begin{gathered}
\int_{0}^{1}\left[f-\sum_{0}^{N}\left(\alpha_{i}+\beta_{i} h_{i}\right) K_{i}\right] K_{m} d x=0, \\
\int_{0}^{1}\left[f-\sum_{0}^{N}\left(\alpha_{i}+\beta_{i} h_{i}\right) K_{i}\right] K_{m} h_{m} d x=0 .
\end{gathered}
$$

Now if $I=\left[a_{i_{m}}, a_{i_{m+1}}\right)$, we obtain from (2) and (3) respectively

and

$$
\int_{I} f d x=\alpha_{m}|I|+\beta_{m} \frac{|I|^{2}}{2}
$$

$$
\int_{I} f h_{m} d x=\alpha_{m} \frac{|I|^{2}}{2}+\beta_{m} \frac{|I|^{3}}{3} .
$$

Thus

and

$$
\alpha_{m}=\frac{2}{|I|^{2}} \int_{I}\left(2|I|-3 h_{m}\right) f d x
$$

Since

$$
\beta_{m}=\frac{6}{|I|^{3}} \int_{I}\left(2 h_{m}-|I|\right) f d x .
$$

and

$$
\int_{I}\left(2|I|-3 h_{m}\right) d x=\frac{|I|^{2}}{2}
$$

we have if $x_{0} \in I$,

$$
\int_{I}\left(2 h_{m}-|I|\right) d x=0,
$$

$$
\begin{aligned}
\left|S_{N}\left(x_{0}, f\right)-f\left(x_{0}\right)\right|= & \left|\alpha_{m}+\beta_{m} h_{m}\left(x_{0}\right)-f\left(x_{0}\right)\right| \\
= & \mid \frac{2}{|I|^{2}} \int_{I}\left[2|I|-3 h_{m}(x)\right]\left[f(x)-f\left(x_{0}\right)\right] d x \\
& +\frac{6 h_{m}\left(x_{0}\right)}{|I|^{3}} \int_{I}\left[2 h_{m}(x)-|I|\right]\left[f(x)-f\left(x_{0}\right)\right] d x \mid
\end{aligned}
$$


If $x_{0}$ is a point of continuity of $f$, there exists a positive number $\delta$ such that $\left|f(x)-f\left(x_{0}\right)\right|<\varepsilon$ when $x \in I$ and $|I|<\delta$. By Lemma 1 there is an integer $N_{\delta}$ such that if $N>N_{\delta},|I|<\delta$ and since $\left|h_{m}(x)\right| \leqq|I|$ when $x \in I$, we find from (6) if $N>N_{\delta}$,

$$
\left|S_{N}\left(x_{0}, f\right)-f\left(x_{0}\right)\right|<10 \varepsilon+18 \varepsilon=28 \varepsilon .
$$

THEOREM 3. If $f$ is continuous on $[0,1]$, the Fourier $-u_{n}$ expansion (1) converges uniformly to $f(x)$ on $[0,1]$.

Proof. If $\varepsilon>0$, there exists a $\delta>0$ such that $\left|f\left(x_{1}\right)-f\left(x_{2}\right)\right|<\varepsilon / 28$ when $x_{1}, x_{2}$ $\in[0,1]$ and $\left|x_{1}-x_{2}\right|<\delta$. By Lemma 1 we can choose an integer $N_{\delta}$ such that if $N>N_{\delta}$, the norm of the partition of $[0,1]$ determined by $A_{N}$ is less than $\delta$. Then from equation (6) of the preceding proof we see that $\left|S_{N}\left(x_{0}, f\right)-f\left(x_{0}\right)\right|<\varepsilon$ for any $x_{0} \in[0,1]$.

In closing it should be pointed out that if the set $A$ involved in the definition of $\left\{u_{n}\right\}$ is taken to be the particular set described in $\S 7(\mathrm{~B})$ of [2], the resulting $\left\{u_{n}\right\}$ is the orthonormal sequence of functions defined by Franklin [1].

\section{REFERENCES}

1. P. Franklin, A set of continuous orthogonal functions, Math. Ann. 100 (1928), 522-529.

2. J. L. Sox and W. J. Harrington, A class of complete orthogonal sequences of step functions, Trans. Amer. Math. Soc. 157 (1971), 129-135.

Department of Mathematics, North Carolina State University, Raleigh, North Carolina 27607 